\title{
Industrial wastewater treatment by means of membrane techniques
}

\author{
Maria Tomaszewska \\ Szczecin University of Technology, Institute of Chemical and Environment Engineering, Pułaskiego 10, 70-322 Szczecin, \\ Poland, e-mail: maria.tomaszewska@ps.pl
}

\begin{abstract}
The importance of membrane processes in wastewater treatment is continuously growing. Membranes can be used for the separation of liquids, dissolved or suspended solids or solutes in the colloid form. The main features of membrane processes application for the treatment of spent solutions are possibilities of removal or recovery of valuable or harmful components as well as the possibility of closing water systems what reduce fresh water consumption. Very often the use of membrane processes allow to purify wastewater to a degree difficult to achieve by conventional techniques. The possibility of an application of pressure driven techniques such as ultrafiltration (UF), nanofiltration (NF) and reverse osmosis (RO) for oily wastewater treatment, has been presented. The studies on the two-stage treatment of bilge water combining UF and RO or NF have demonstrated a high effectiveness of purification. NF could also be applied to recover glycols from spent coolant liquids. The utilization of membrane distillation for saline wastewater concentration or for the treatment of spent metal pickling solutions was demonstrated.

The potential application of a hybrid process combining photocatalytic degradation of organic matter with membrane separation for the treatment of wastewaters from textile industry has been shown.
\end{abstract}

Keywords: membrane techniques, wastewater treatment, industrial wastewater.

Presented at VII Conference Wasteless Technologies and Waste Management in Chemical Industry and Agriculture, Międzyzdroje, 12 - 15 June, 2007.

\section{INTRODUCTION}

Membrane processes are rather still considered as a new method of separation. However, membranes present such various structures and separation possibilities that an application of membrane processes is still growing. Membranes can be used for the separation of mixture of gases, vapors, liquids, dissolved or suspended solids or solutes in the colloid form. The driving forces of mass transport through a membrane can have different character - the difference of concentration, pressure, partial pressure, temperature or electrical potential. The combination of a membrane with proper characteristics and appropriate driving force creates the family of membrane processes allowing to obtain satisfactory results of the separation of mixture components. The possibilities of the recovery of valuable components or dangerous for the environment make membrane processes an interesting alternative to the conventional methods of wastewater treatment. Moreover, membrane filtration in comparison with the conventional techniques offers many benefits - separation can be carried out continuously and under mild conditions, energy consumption is generally low, up-scaling is easy. Recently, membrane processes have often been combined with other techniques to obtain higher separation efficiency. Membrane bioreactors or reactors comprise the new family of the advanced membrane application.

The pressure driven techniques such as reverse osmosis (RO), nano-, ultra- and microfiltration (NF, UF, MF) have recently attracted considerable attention as the methods for wastewater treatment. The driving force for mass transfer across a membrane is realized by a difference of the transmembrane pressure. The processes differ in the extent of this pressure difference and the type of the membrane employed. The RO and NF membranes are nonporous with sorption and diffusion as the transport mechanism. Because of the more dense structure of the RO membranes, higher pressure on the feed side is applied, in the range of $6.0-10 \mathrm{MPa}$, sometimes even $20 \mathrm{MPa}^{1}$. Moreover, the sieving mechanism is responsible for the rejection of uncharged molecules ( $>200 \mathrm{Da}$ ) by NF membrane and the Donnan exclusion mechanism influences the rejection of the charged species. Most of NF membranes contain charged functional groups, therefore the electrostatic interaction between the charged solute species and the membrane surface is crucial, for the transfer or the rejection of ions ${ }^{2}$. RO and NF can be used for desalination, the removal of petroleum derivatives from oily wastewater as well as to remove the organic substances from wastewater. The divalent or higher valent salts can be separated from the monovalent salts using the NF method.

The RO and NF membranes are very sensitive to fouling, decreasing a resultant permeate flux, therefore the MF or UF can be employed as a pretreatment method. The UF and MF membranes are porous and have been applied for the separation of macromolecules, suspensions, colloids. The UF process is carried out under a lower pressure difference than for the RO or NF, typically in the range of $0.2-0.7$ (sometimes up to 1.0) MPa.

The electrically driven processes in which electrical potential difference acts as a driving force creates group processes using ion exchange membranes. The processes involve monopolar electrodialysis, bipolar electrodialysis and other electrodialysis (ED)-related processes such as diffusion dialysis (DD), and Donnan dialysis. These processes can be used for the desalination, to remove ions from an aqueous solutions containing organic matter. Electrodialysis is especially recommended for the desalination of a brackish mine waters and the desalination of a number of solutions produced by food industry.

The membrane distillation (MD) process is considered as a new, interesting method which could be applied to 
high purity water preparation as well as to wastewater treatment. The MD driving force is supplied by a partial pressure difference across a microporous hydrophobic membrane caused by a difference of temperatures and compositions of layers adjacent to the membrane. The separation mechanism is based on vapour/liquid equilibrium of a liquid mixture which controls the process selectivity. The MD process is recommended especially for the obtaining of fresh water from the sea and the high purity water for special purposes ${ }^{3}$. However, MD could be applied for the desalination and to the concentration of solutions of non-volatile salts close to the supersaturated state $^{4}$, as well as to the acids solutions concentration ${ }^{5}$. In the case of volatile compounds, they can be removed from spent solutions ${ }^{6}$. Owing to the low temperature of the processed solutions MD could be an attractive method of the concentration of temperature sensitive substances. A low operation temperature, considerably lower than the boiling point of the feed enables the utilization of the low grade or an alternative energy sources.

\section{EXAMPLES OF MEMBRANE PROCESSES APPLICA- TION FOR THE INDUSTRIAL WASTEWATER TREAT- MENT}

\section{Recovery of metals from dilute waste}

Wastewater produced by metal finishing industries very often contains harmful heavy metals. The components from the spent liquors had to be removed, recovered or recycled for environmental reasons. The conventional methods such as neutralization or chemical precipitation is not always sufficient for their purification. Moreover, the possibilities for the recovery of both toxic and valuable metals should first be evaluated.

In recent years the use of membrane processes in the recovery of metals from dilute spent liquors has increased. The main area of application are rinse waters from electroplating industry or spent pickling liquors or even the galvanizing bath. Membrane techniques such as RO, NF, ED, DD allow the separation of metal ions from waste solutions and recover both metals and water. The removal of metal ions from electroplating rinse water by electrodialysis is especially attractive, because the concentrated solution can be recycled directly into the galvanizing tank, whereas the water can be recycled to rinsing. The ED process was successfully applied for the recovery of $\mathrm{Ni}$, $\mathrm{Cu}, \mathrm{Au}, \mathrm{Cd}, \mathrm{Ag}, \mathrm{Pd}, \mathrm{Cr}^{7}$. Reverse osmosis (RO) or nanofiltration (NF) is the other method which could be applied for the treatment of electroplating solutions. The liquors containing metals may be concentrated and the obtained streams of retentate and pure water can be recycled, which allows closing water systems, reducing fresh water consumption. However, the membrane used in RO or NF are sensitive to the extreme values of $\mathrm{pH}$. The processes can be successfully applied in connection with any different techniques.

The hybrid complexation-ultrafiltration process is an attractive method of the removal of metal ions from industrial wastewater. The metal ions are bound by a polymer ligand into compounds with the size of molecules which allow to retentate them by ultra- or microfiltration. The polymer-metal complex is then decomposed (e.g. changing the $\mathrm{pH}$ ) to recover the complexing agent. The solution containing the concentrated metal ions can be separated using subsequent UF. Various polymers can be used as a complexing agent - poliethyleneimine, sodium polyacrylane. High efficiency of metal recovery, up to $92 \%$ was achieved ${ }^{8}$.

\section{Treatment of oily wastewater}

Oily waste water can be generated by the metal industry, during cutting, drilling or grinding of metal components. The oily spent solution can be also formed in the case of the plant manufacturing metal wires (cables) for the electrical industry, or by floating objects, ships in the engine rooms (bilge water). Bilge water constitutes about $20 \%$ of oily wastewater discharged from ships into the sea. The spent emulsions depending on the place of their generation can contain oils, lubricants, metal ions as the major components as well as wetting agents, corrosion inhibitors or emulsifiers. The limits for oil content in the wastes discharged into the environment or prior to further treatment caused that the emulsions have to be split to separate the oil from the water phase. The recovered oil may be used as a fuel or as a hydraulic fluid. However, the aqueous phase still contains too much oil, additives and metal ions. Further treatment of the water is required before it could be discharged. The traditional methods used worldwide for the treatment of the oily wastewater obey coalescence, coagulation or filtration and further biological methods.

The membrane technologies such ultrafiltration, nanofiltration and RO or their combinations are a modern alternative to the conventional methods. During the membrane technology treatment the feeding solution is divided into the permeate, which comprises the stream passing through a membrane and the remaining stream retentate. The composition of the permeate is influenced by the membrane structure and their permeability under operation conditions of the applied membrane techniques.

During ultrafiltration the membrane forms a barrier for large particles and small oil droplets. It was found that UF with the membrane with the molecular weight cut-off (MWCO) equal to $100 \mathrm{kDa}$ can effectively reduce the suspended solids, oil and lubricants. The permeate with the oil content below 10 ppm was achieved. The rejection of the turbidity and suspended solids exceeded $99 . \% 9$. The resultant permeate complies with the standards concerning the discharge of oily water into the environment.

However, the ionic components of the wastewater and organic matter with low molecular weight were rejected by UF only in $10-30 \%$. Then, for further deeper purification, the nanofiltration or reverse osmosis can be proposed as a second step of the wastewater treatment, Fig. 1. When the UF/NF system was applied to a spent cooper wire drawing emulsion treatment the rejection of the copper ions was at the level of $92 \%$. In the case of the combination of UF and RO, where the UF permeate was used as a feed of RO, a high degree of rejection of both inorganic species and the organic compound (analyzed as TOC) was achieved, higher than $99.5 \%$.

A bilge water purification in the combination of UF/NF and UF/RO (Fig.2) also exhibited high effectiveness. During the first step of the treatment ( the UF tubular module equipped with FP 100 membrane) oil was re- 


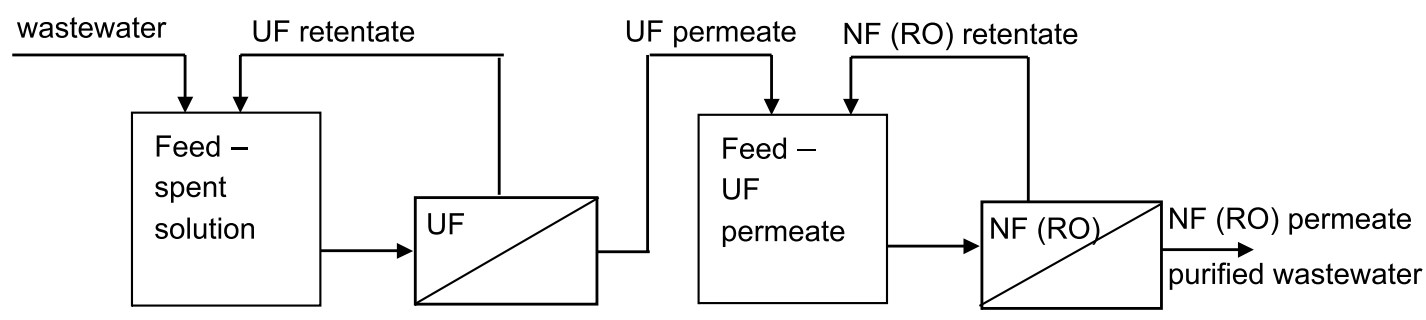

Figure 1. The flow sheet diagram of wastewater treatment using the combination of UF and NF (RO)

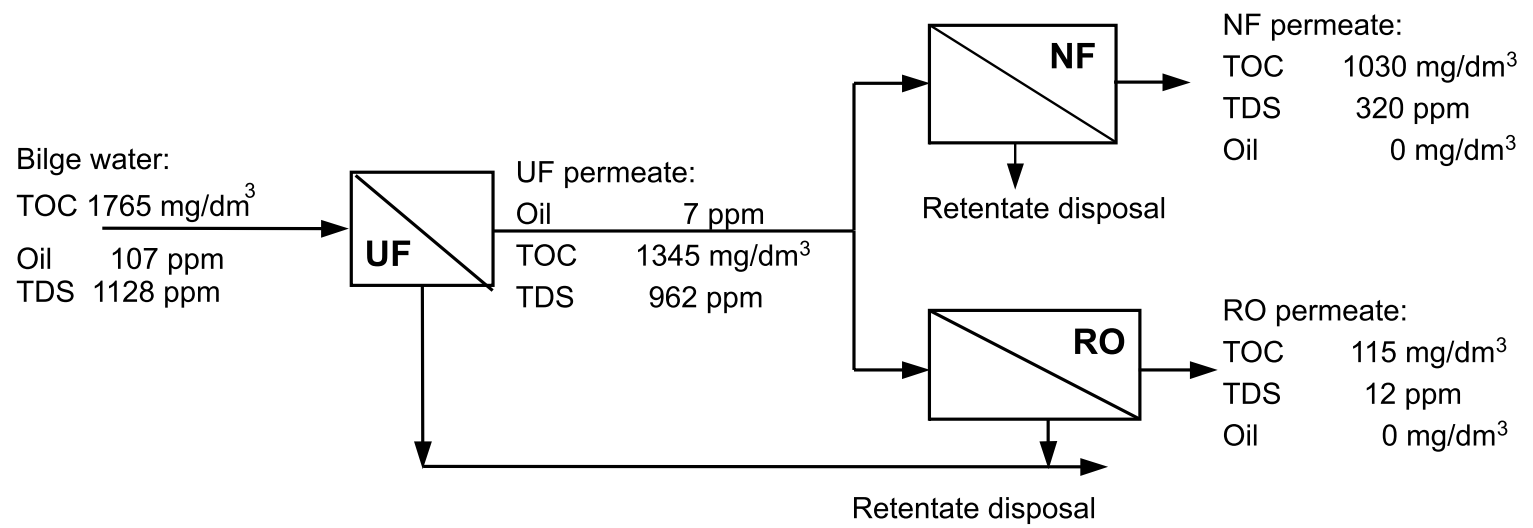

Figure 2. The scheme of bilge water treatment using a combination of UF and NF or UF and RO

moved in $95 \%$, whereas the application NF or RO as a second step allow a complete removal of oil. Rejection of organic compounds (measured as TOC) in NF using the NF AFC30 membrane was equal to $71.5 \%$. As a result of RO (membrane BW 3040) application as a second stage of bilge water treatment the organic matter was removed in $99.4 \%$. During these studies the UF permeate flux for pressure $0.2 \mathrm{MPa}$ was equal to $21 \mathrm{dm}^{3} / \mathrm{m}^{2} \mathrm{~h}$, whereas for $\mathrm{NF}(\Delta \mathrm{P}=0.5 \mathrm{MPa})$ and $\mathrm{RO}(\Delta \mathrm{P}=3.0 \mathrm{MPa})$ were 23 and $35 \mathrm{dm}^{3} / \mathrm{m}^{2} \mathrm{~h}$, respectively ${ }^{11,12}$.

The UF/RO combination exhibited high effectiveness. The resultant permeate purity was so high that it could be directly reused for fresh emulsion preparation or in other industrial applications.

\section{Recovery of ethylene glycol}

Ethylene glycol (EG) is employed in most of the commercial coolant liquids (a mixture of water and glycol) commonly applied in vehicles like cars or buses. However, EG is considered as a harmful agent, therefore direct discharge of the spent coolant liquids into environment is prohibited in many countries. Moreover, the spent liquor contained besides EG also nitrosoamines, heavy metals and mineral oils ${ }^{13}$. Studies of EG separation were performed using membrane distillation. During the process all non-volatile compounds together with EG were concentrated on a feed side.

However, it was found that EG passes through a nanofiltration membrane, hence it could be recovered on a permeate side, whereas the inorganic contaminants would be rejected. The experiments performed with model solutions have shown that the degree of the passage of EG was affected by the content of EG in the feed. In the case of the NF AFC30 membrane the degree of passage from 90 to $80 \%$ was achieved for EG concentrations in the range of 1 to $20 \mathrm{wt} \%$, respectively. The rejection of TDS was higher than $95 \%$, which was presented in Fig. $3^{14}$.

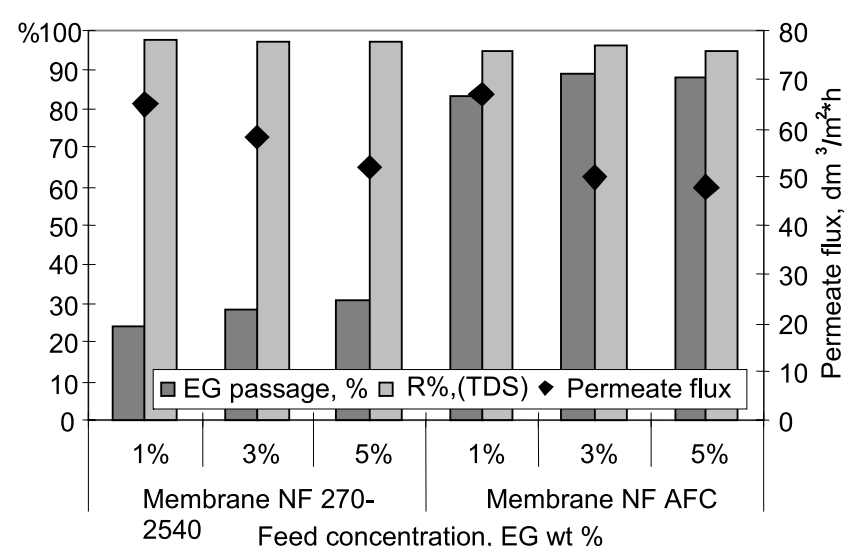

Figure 3. The efficiency of EG recovery from model solutions using nanofiltration

The studies demonstrated high effectiveness of EG recovery using nanofiltration. The resultant permeate, containing ethylene glycol could be reused for the production of a coolant liquid.

\section{The concentration and recovery of mineral acids}

In industry huge volumes of diluted mineral acids which could be concentrated and reused, are generated. Membranes applied in membrane distillation are chemically and thermally resistant, therefore MD could be employed for these purposes. Studies performed in our laboratory showed that sulfuric and phosphoric acids can be concentrated to ca. $63 \%$ (at a feed temperature equal to $333 \mathrm{~K}$ ) and $54 \% \mathrm{P}_{2} \mathrm{O}_{5}$. Both acids are non-volatile, therefore they were completely retained on the feed side. The concentration of the sulfuric acid solution obtained after apatite phosphogypsum extraction used to recover lanthane compounds, was performed employing membrane distillation ${ }^{4}$. As it was described earlier the results of the studies were very interesting, hence the novel technology being an alternative to evaporators was proposed . 
Various acids are used for the cleaning of metal elements before electroplating. The spent pickling solution contains residual acids and salts of frequently harmful heavy metals. The neutralization procedure commonly used for the effluent treatment is not always sufficient. Moreover, taking environment protection into account, the recovery of valuable or toxic components from the effluents before their disposal is a better way. Electrodialysis was proposed for the recovery of hydrochloric or sulfuric acids from the spent solutions. The results of our work have shown that hydrochloric acid could be successfully separated from the spent pickling solutions by membrane distillation ${ }^{5}$. Hydrochloric acid is a volatile substance, therefore it was transferred through a membrane together with the water vapour at MD conditions, whereas salt as non-volatile was retained on a feed side. The experiments were performed with a raw metal pickling solution taken from an electroplating plant. A gradual concentration of the liquor using MD was conducted up to the supersaturated state. The solid residue in the feed after the concentration achieved $600 \mathrm{~g} / \mathrm{dm}^{3}$. The pure hydrochloric acid obtained on the distillate side achieved the concentration of $150 \mathrm{~g} / \mathrm{dm}^{3}$. It could be recycled directly for the preparation of the fresh etching solution or after further concentration - Fig.4. The pure water obtained at the beginning of the concentration of the acid could be applied for rinsing metal object from the etching bath. The separated salts of cuprum or iron may be utilized or restored.

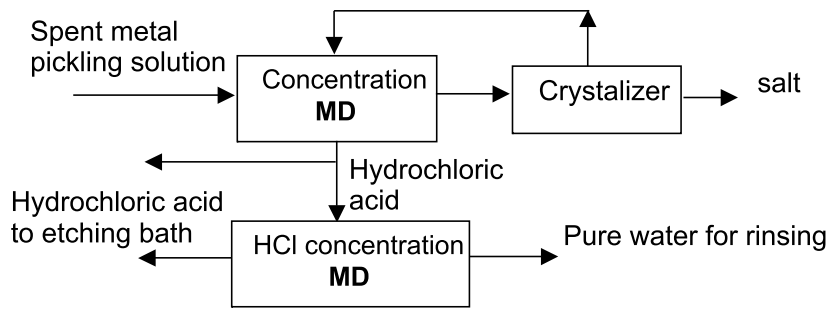

Figure 4. The flow sheet diagram of the treatment of spent metal pickling solution using membrane distillation

The treatment of wastewater produced during the regeneration of ion exchangers demonstrated that the application of appropriate pretreatment is necessary to remove foulants. The MD process applied for such wastewater treatment allowed to obtain pure water of the electrical conductivity amounting to $2-4 \mu \mathrm{mS} / \mathrm{cm}^{15}$.

\section{Textile wastewater treatment}

A dying process of textiles is characterized by high water consumption, caused by the multiple washing and rinsing cycle. Thus, a significant amount of coloured wastewater with high COD is generated. The conventional methods of the wastewater treatment use adsorption, biological degradation, chlorination or ozonation. Combination of UF with RO or MD permits to obtain a concentrate which may be recycled to the dying bath and pure water used for washing cloths after the dying process.

The novel method of the treatment of coloured wastewater is a combination of photocatalytic degradation of organic matter under UV or visible light radiation and membrane technique, Figure 5. The membrane in the photocatalytic membrane reactor (various configuration) plays both the role of a barrier for the catalyst and the selective barrier for the molecules to be degraded and the products of their decomposition. Photocatalytic degradation of azo-dye was curried out using $\mathrm{TiO}_{2}$ as a photocatalyst. Ultrafiltration or membrane distillation may be applied to $\mathrm{TiO}_{2}$ separation and the dye ${ }^{16}$. The results show that the efficiency of dye removal and the products of its decomposition was very high. In the case of the combination with membrane distillation the dye was completely removed, TOC for $73 \%$ and TDS for $93 \%$.

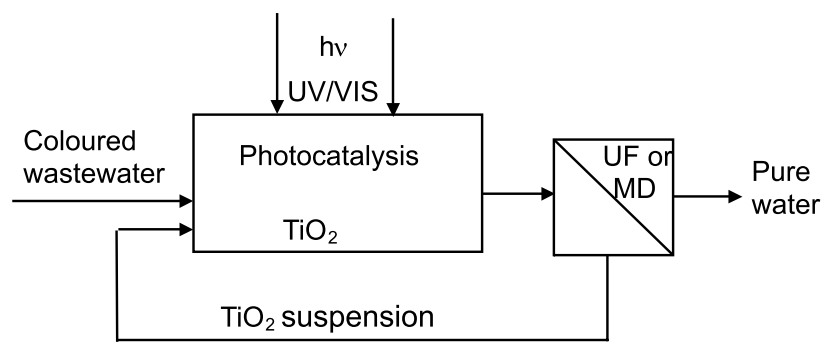

Figure 5. The flow sheet diagram of the treatment of a model solution containing azo-dye using the photocatalytic membrane reactor

\section{CONCLUSIONS}

Membrane techniques are very promising methods of wastewater treatment. The use of membrane processes allow to purify wastewater very often to a degree difficult to achieve by conventional techniques.

\section{LITERATURE CITED}

(1) Rautenbach R., Mellis R.: Desalination, Hybrid processes involving membranes for the treatment of highly organic/inorganic contaminated waste water, 1995, 101, 105113.

(2) Nanofiltration- Principles and Applications, ed by Schafer A. I., Fane A. G., Waite T. D., Elsevier Ltd, 2005.

(3) Lawson K. W., Lloyd D. R.: Membrane distillation, J. Membrane Sci., 1997, 124, 1 - 25.

(4) Tomaszewska M.: Concentration of the extraction fluid from sulfuric acid treatment of phosphogypsum by membrane distillation, J. Membrane Sci., 1993, 78, 277 - 282.

(5) Tomaszewska M., Gryta M., Morawski A. W.: Study on the concentration of acids by membrane distillation, J. Membrane Sci., 1995, 102, 113 - 122.

(6) Tomaszewska M., Gryta M., Morawski A. W.: Recovery of hydrochloric acid from metal pickling solutions by membrane distillation, Sep.Pur.Tech., 22 - 23 (2001) 591 - 600.

(7) Scott K.: Handbook of Industrial Membranes, Elsevier Ltd, 1997.

(8) Bodzek M., Korus I., Loska K.: Application of hybrid complexation-ultrafiltration process for removal of metal ions from galvanic wastewater, Desalination, 121, 1999, 117 $-121$.

(9) Karakulski K., Morawski A. W.: Purification of copper wire drawing emulsion by application of UF and RO, Desalination, 2000, 131, 87 - 95.

(10) Karakulski K., Morawski A. W.: Treatment of spent emulsion from a cable factory by an integrated UF/NF membrane system, Desalination, 2002, 149, 163 - 167.

(11) Tomaszewska M.: Studies on the application of membrane technology in chemical industry, Polish Journal of Chemical Technology, 2005, 7 (3), 101 - 107.

(12) Tomaszewska M., Orecki A., Karakulski K.: Treatment of bilge water using combination of ultrafiltration and reverse osmosis, Desalination, 2005, 185, 1629 - 1638. 
(13) Rincon C., Ortiz de Zarate J. M., Mengual J. I.: Separation of water and glycols by direct contact membrane distillation, J.Membrane Sci., 1999, 158, 155 - 165.

(14) Orecki A., Tomaszewska M., Karakulski K., Morawski A. W.: Separation of ethylene glycol from model wastewater by nanofiltration, Desalination, 2006, 200, 358 - 360 .

(15) Gryta M., Tomaszewska M., Karakulski K.: Wastewater treatment by membrane distillation, Desalination, 2006, 198, $67-73$.

(16) Mozia S., Tomaszewska M., Morawski A. W.: Removal of azo-dye Acid red 18 in two hybrid membrane systems employing a photodegradation process, Desalination, 2006, 198, $183-190$. 